\title{
Reliable elimination of telluric lines from stellar spectra ${ }^{\star}$
}

\author{
M. Hrudková ${ }^{1}$ and P. Harmanec ${ }^{1,2}$ \\ 1 Astronomical Institute of the Charles University, V Holešovičkách 2, 18000 Praha 8, Czech Republic \\ e-mail: mary@atrey.karlin.mff.cuni.cz \\ 2 Astronomical Institute, Academy of Sciences, 25165 Ondřejov, Czech Republic
}

Received 24 January 2005 / Accepted 27 February 2005

\begin{abstract}
We demonstrate that the powerful disentangling technique used to separate the individual spectra of binary components and telluric lines can also be used to remove telluric lines from the spectrum of a single star by treating the telluric spectrum as a second star. We tested that on the spectra of $\alpha$ Boo (=Arcturus) secured with a Reticon detector in the coudé focus of the Ondřejov 2-m telescope. We demonstrate that the method works well and is invaluable especially for the spectra of red stars which are heavily blended with the telluric lines. The disadvantage of the method is that one needs a number of spectra of the star in question, secured at different months of the year. The advantage is that one obtains the final disentangled stellar spectrum with a high $S / N$ ratio.
\end{abstract}

Key words. line: profiles - techniques: spectroscopic - stars: individual: $\alpha$ Boo

\section{Introduction}

One serious problem that complicates the interpretation and quantitative analysis of stellar spectra is the presence of numerous telluric lines in the red and infrared parts of the electromagnetic spectrum. At the same time, those regions contain some astrophysically important stellar lines.

The blending is especially acute for stars of later spectral classes which have numerous stellar lines and usually low projected rotational velocities. A technique that is often used to remove the telluric lines from the stellar spectrum is to divide the observed spectrum by the (more or less featureless) spectrum of a blue star. The obvious disadvantage of this procedure is that the strength of the telluric lines varies both with time and the position in the sky. Therefore, the removal of telluric lines is not perfect and one has to invest non-negligible additional observing time to obtain spectra of blue stars at the airmasses very similar to the instantaneous airmass of the program star and shortly before or after its exposure.

Hadrava $(1995,1997)$ developed the program KOREL, a powerful technique of spectral disentangling of component spectra in binary and multiple systems of stars ${ }^{1}$. An assumption on which his method is based is that the spectral line profiles are not variable in shape but only in relative intensity from

\footnotetext{
* Based on spectra from the Ondřejov Observatory.

1 The program KOREL is made freely available to interested users by the author. Its more detailed description, Fortran source code and user's guide can be obtained via

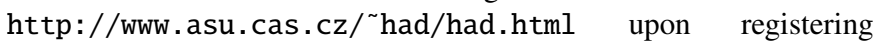
yourself athad@sunstel.asu.cas.cz.
}

one spectrum to another. The disentangling works because the component spectra vary in wavelength as the stars move around their common centre of gravity. Hadrava also included the possibility of disentangling telluric lines that are modelled as a distant companion moving in an orbit with a period of one tropical year and an amplitude that depends on the stellar coordinates. Since KOREL can model and derive the varying line strengths (see Hadrava 1997), it is well suited to the disentangling of telluric lines.

The success of KOREL in disentangling the stellar and telluric lines was first demonstrated by Harmanec et al. (1997) for V436 Per, a binary consisting of two similar B stars.

We realized that the same principle should also be applicable to an objective removal of telluric lines from the stellar spectrum of a single star if one deals with it as if it would be a binary in orbit with a nearly zero velocity amplitude and models the telluric lines via the motion of the Earth around the Sun in the usual way.

To test the feasibility of the method, we decided to use spectra of the bright red giant and a radial-velocity (RV hereafter) standard $\alpha$ Boo (Arcturus, HD 124897) which can be observed for a large fraction of the year at a latitude of about $50^{\circ}$. A number of Reticon spectra of $\alpha$ Boo were accumulated in the Ondřejov Observatory archive between 1992 and 1998.

Arcturus has been classified as a red giant (spectral type K1.5 III; Keenan \& McNeil 1989). The SIMBAD database gives a RV $=-5.2 \mathrm{~km} \mathrm{~s}^{-1}$. $\alpha$ Boo is now known to exhibit small RV and also light variations. The situation is not entirely clear since different authors have published 
different low-amplitude RV curves with a number of different periods and amplitudes. First, Campbell et al. (1982) suggested that Arcturus was a RV variable based on data separated by a two-day interval. Smith et al. (1987) not only confirmed the Arcturus radial-velocity variability but also reported the presence of a 1.84 period with an amplitude of $0.2 \mathrm{~km} \mathrm{~s}^{-1}$. Cochran (1988) then confirmed their period. Irwin et al. (1989) confirmed this short-term variability and demonstrated a long-term variability with the largest amplitude component having a period of 640 days or more. Later Belmonte et al. $(1990 \mathrm{a}, \mathrm{b})$ reported the presence of 2.7 and $8.3 \mathrm{RV}$ periods. Other short periods of RV variations were reported by Hatzes \& Cochran (1994), namely about 2.46 and 4.0, both with amplitudes of $0.1 \mathrm{~km} \mathrm{~s}^{-1}$. In an earlier study, the same authors also concluded that besides the rapid RV changes there are also long-period RV variations with periods of about 233 days and an amplitude of $0.5 \mathrm{~km} \mathrm{~s}^{-1}$, and 46 days with a much smaller RV amplitude of only $0.05 \mathrm{~km} \mathrm{~s}^{-1}$ (Hatzes \& Cochran 1993). These variations were invariably interpreted as evidence of radial pulsations. Retter et al. (2003) concluded that the oscillations of Arcturus could be caused by sound waves, but it is not clear whether these are coherent p-mode oscillations or perhaps a single mode with a short lifetime. The latter would explain the different periods found by various authors. Only longer time observations can distinguish between the two options.

However, it is obvious that the RV variations are so small that it is safe to treat $\alpha$ Boo as a star with constant RV for our purpose.

\section{Reduction of the data}

Observations of $\alpha$ Boo were obtained between August 1993 and May 1998. They have a good phase distribution over the whole year. We have processed 50 Reticon spectra from the Ondřejov coudé spectrograph with good $S / N$. All these spectra have a linear dispersion of $17.2 \mathrm{~A} \mathrm{~mm}^{-1}$ and a two-pixel resolution of about 12600 . They cover the wavelength region 6300-6700 ̊.

All spectra were flatfielded, wavelength calibrated and rectified with the help of the program SPEFO - see Horn et al. (1996) and Škoda (1996). The barycentric corrections of the radial velocity were added, based on the program BRVEL by Drs. S. Yang and J. Amor, kindly put at our disposal by Dr. S. Yang. Then, an auxiliary program PREKOR (provided by Dr. P. Hadrava along with KOREL) was used to prepare the input data for KOREL. PREKOR allows one to extract a userdefined spectral region from a set of input spectra and interpolate them into the equidistant logarithmic wavelength scale required by KOREL.

\section{Disentangling the stellar and telluric lines}

As schematically shown in Fig. 1, redrawn after Hadrava (1995), KOREL is designed to disentangle spectra of up to 5 components of a hierarchical system of two binary pairs (components 1 and 2, and 3 and 4, respectively) which move

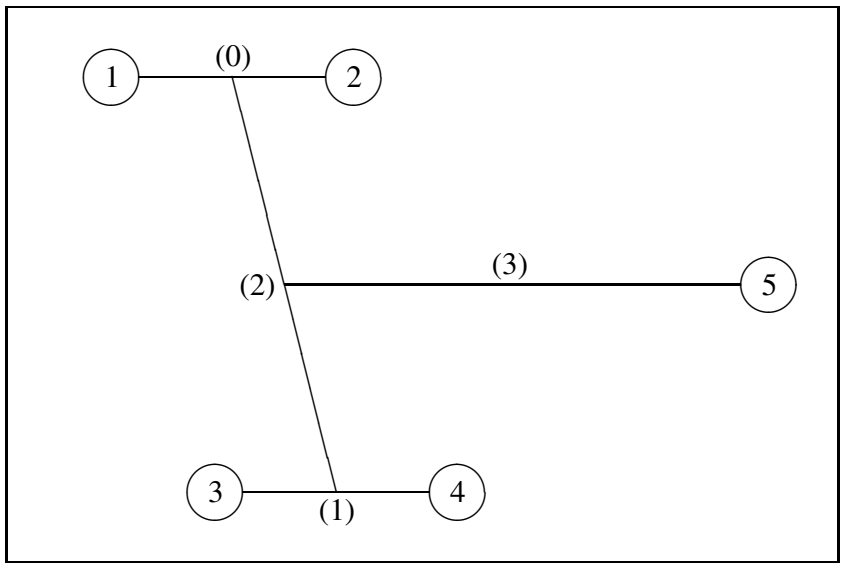

Fig. 1. The hierarchical structure of the stellar system for KOREL.

around their local centres of gravity in orbits Nos. 0 and 1, respectively, and also with respect to each other around the common centre of gravity in orbit No. 2. There can also be a distant component 5 which revolves in an orbit No. 3 around the common centre of gravity with the quadruple system. In our particular case, the situation is simplified to only two spectra. The stellar spectrum is treated as component 1 of a "binary" system with a negligible RV amplitude. (In our application to $\alpha$ Boo see below - we used $K_{1}=0.001 \mathrm{~km} \mathrm{~s}^{-1}$ and set the mass ratio $M_{2} / M_{1}=1$ for simplicity). The auxiliary program PREKOR also provides the parameters for the distant fifth component identified with the Earth moving in the orbit around the Sun. In particular, the semiamplitude of the RV variations of the putative quadruple system moving around the common centre of gravity is set equal to $0.001 \mathrm{~km} \mathrm{~s}^{-1}$ and the mass ratio is derived in such a way as to ensure that the semiamplitude of component 5 corresponds to the projected RV of the Earth in the direction towards the object in question. All other parameters (orbital period, eccentricity, longitude of periastron and the periastron advance) are set equal to the known parameters of the Earth's orbit and kept fixed during the solutions. In other words, KOREL is only used to disentangle the stellar and telluric spectrum, without any allowance for convergence of any real or fictitious orbital parameters. We only allowed convergence of line strengths of the telluric lines in each spectrum.

\section{Application to $\alpha$ Boo}

For the test calculations, we selected the spectral region 6442.0-6603.5 ^ which contains a number of telluric water vapour lines. To achieve a better resolution, we divided the selected wavelength interval into 3 subsets and allowed KOREL to disentagle these three data subsets in one solution. Note, however, that in all figures we show the spectra for the whole spectral range covered by those three subsets.

In Fig. 2, a single observed Reticon spectrum of $\alpha$ Boo (taken on HJD 2449562.3466 ) is compared to the KOREL disentangled spectrum of $\alpha$ Boo which is free of any telluric lines. It is seen that the observed and disentangled spectra differ from each other in the presence of the telluric lines in the observed spectrum. To demonstrate that it is indeed so, we compare 


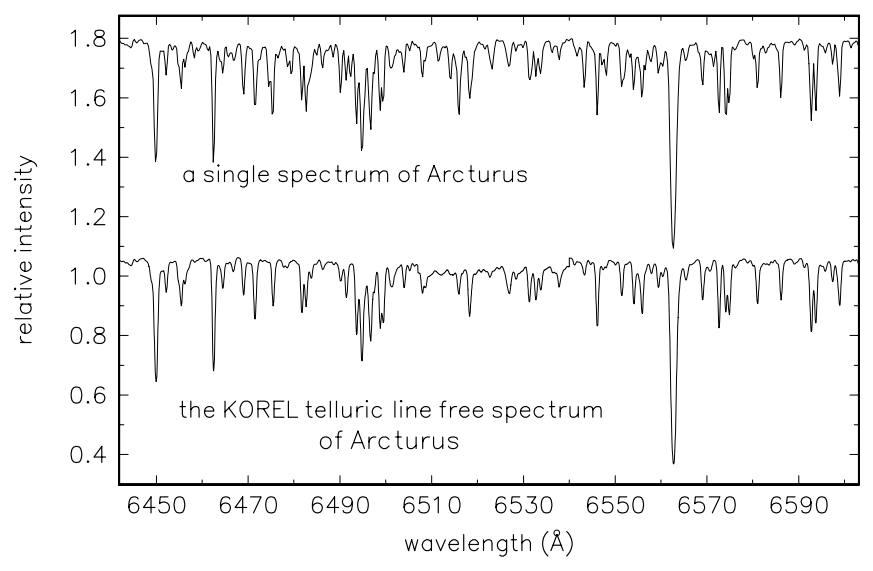

Fig. 2. The disentangled spectrum of $\alpha$ Boo from the KOREL solution compared with a single observed Reticon spectrum of $\alpha$ Boo (taken on HJD 2449 562.3466).



Fig. 3. The disentangled spectrum of telluric lines from the KOREL solution compared to the differential spectrum of two spectra from Fig. 2, i.e. the observed minus KOREL-disentangled Arcturus spectrum.

the difference between the observed and disentangled spectrum with the KOREL disentangled spectrum of telluric lines - see Fig. 3. There is a very good correspondence between the two.

In Fig. 4, two observed Reticon spectra of $\alpha$ Boo are compared to the KOREL disentangled spectrum of telluric lines. The two observed Reticon spectra were taken on HJD 2450152.5704 and 2450252.3465 . They were chosen in order to have a large difference between the barycentric RV corrections. One can see better how the telluric lines affect the two observed spectra. Note that one would barely note the presence of most of the telluric lines in the observed spectra of $\alpha$ Boo (cf. Fig. 2) because of the heavy blending with the stellar lines and similarity in their width.

In Fig. 5 we compare the disentangled spectrum of telluric lines with the spectrum of a blue star $\eta$ UMa obtained with the same Reticon detector, in which the telluric lines are directly visible.

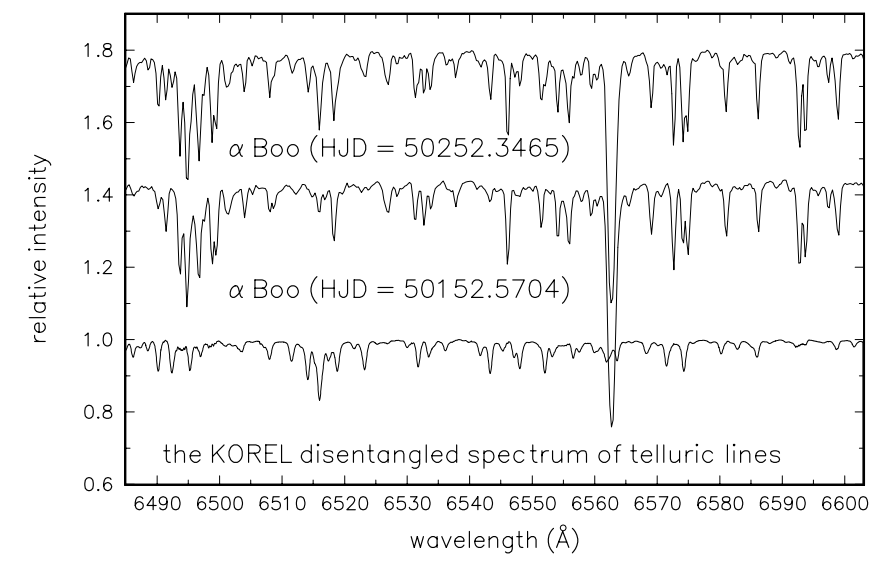

Fig. 4. The disentangled spectrum of telluric lines from the KOREL solution applied to $\alpha$ Boo spectra is compared to two observed Reticon spectra of $\alpha$ Boo, having widely different barycentric $\mathrm{RV}$ corrections.

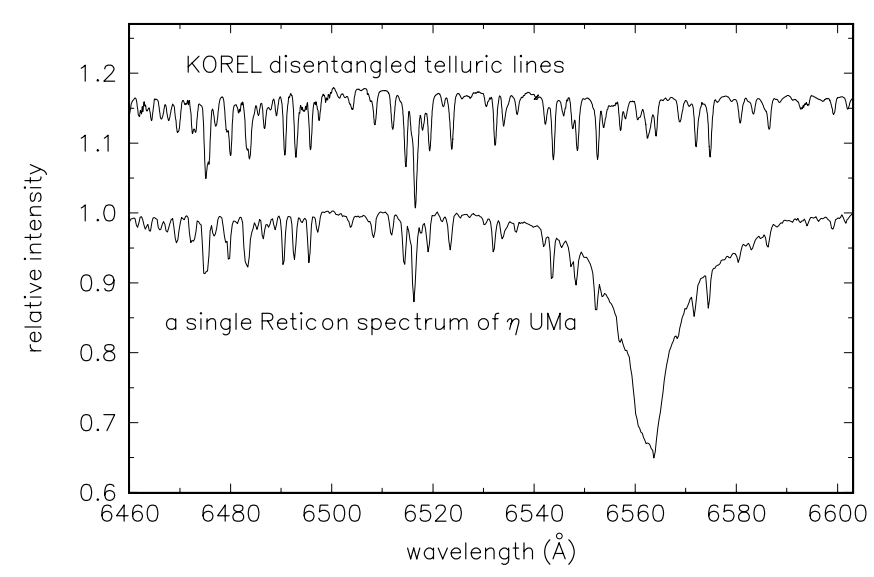

Fig. 5. The disentangled spectrum of telluric lines from the KOREL solution applied to $\alpha$ Boo spectra compared with one observed Reticon spectrum of a blue star $\eta \mathrm{UMa}$.

It is seen that disentangling returned the telluric line spectrum in very good agreement with the independently observed one.

\section{Limits of applicability of the technique}

As pointed out by Hensberge (pers. com.), there is one principal problem when KOREL is used to disentangle the telluric lines. Since the telluric spectrum has no continuum and represents only additional absorption of the stellar light, using one value for the relative strength of all telluric lines in a given spectrogram is not entirely correct. The strength of the same telluric line which absorbs at stellar continuum would be different if the same line would be absorbing in the core of a strong stellar line. Let us denote $s_{*}(\lambda, t)$ and $s_{\mathrm{t}}(\lambda, t)$ as the depth of a stellar and telluric line at given wavelength $\lambda$ and time $t$. The stellar intensity as a function of wavelength in a rectified spectrum $I_{*}(\lambda, t)$ can be written as

$I_{*}(\lambda, t)=1-s_{*}(\lambda, t)$ 
while the telluric spectrum referred to the stellar continuum is

$$
\begin{aligned}
I_{\mathrm{t}}(\lambda, t) & =1-s_{\mathrm{t}}(\lambda, t) \cdot I_{*}(\lambda, t) \\
& =1-s_{\mathrm{t}}(\lambda, t)+s_{\mathrm{t}}(\lambda, t) \cdot s_{*}(\lambda, t) .
\end{aligned}
$$

While the first two terms of Eq. (2) are formally similar to those of Eq. (1), there is also the third term which is not taken into account during KOREL disentangling. One can easily see that for weaker stellar lines and not very strong telluric lines the contribution of the third term is small. For example, for $s_{*}(\lambda, t)=$ 0.15 and $s_{\mathrm{t}}(\lambda, t)=0.1$, the third term amounts to 0.015 i.e. to 15 per cent of the telluric line strength at the continuum level. If, however, the stellar line is strong, the third term can represent a substantial fraction of $s_{\mathrm{t}}(\lambda, t)$. If KOREL deals with a wavelength region which contains a mixture of weak and strong stellar lines, it probably tries to derive some median line strength, so the actual error is smaller.

It is obvious, however, that one has to be cautious with the disentangling of strong stellar lines. One way out of the problem is to deal with a strong line separately, treating it as one segment of the spectrum in which the telluric line strength is determined separately.

Note also that for accurate work, one can identify the positions of telluric lines using KOREL and then mask these parts in the individual stellar spectra and derive average stellar spectra with the masked parts omitted from the averaging. This should also provide the net stellar spectrum because the telluric lines change their wavelength position from one spectrum to another.

We therefore conclude that the method works well and that with the precautions mentioned it can be used by colleagues studying stellar spectra in the regions where telluric lines appear.

Acknowledgements. We acknowledge the use of programs KOREL and BRVEL kindly put at our disposal by their authors, Drs. P. Hadrava and S. Yang, respectively. The spectra of $\alpha$ Boo were taken by Drs. P. Hadrava, P. Harmanec, D. Holmgren, late J. Horn, P. Koubský, J. Kubát, V. Šimon, P. Škoda, S. Štefl, and M. Wolf while the $\eta$ UMa spectrum was obtained by the late Dr. K. Juza. Also, the use of the SIMBAD database maintained at the Strasbourg Astronomical Data Centre and bibliography from the NASA ADS database is gratefully acknowledged. We profitted from very useful comments on the draft version of this manuscript by Drs. H. Hensberge, D. Holmgren, E. Shkolnik and G.A.H. Walker and by an anonymous referee. This study was partly supported from the research plans J13/98: 113200004 of MŠMT and Z1-003-909 of AV ČR and also from the research project K1-204-3105 of the Academy of Sciences of the Czech Republic and from the grant GA ČR 205/2002/0788 of the Granting Agency of the Czech Republic.

\section{References}

Belmonte, J. A., Jones, A. R., Pallé, P. L., \& Cortes, T. R. 1990a, Ap\&SS, 168, 77

Belmonte, J. A., Jones, A. R., Pallé, P. L., \& Cortes, T. R. 1990b, A\&A, 358, 595

Campbell, B., Irwin, A. W., \& Walker, G. A. H. 1982, BAAS, 14, 625

Cochran, W. D. 1988, ApJ, 334, 349

Hadrava, P. 1995, A\&AS, 114, 393

Hadrava, P. 1997, A\&AS, 122, 581

Harmanec, P., Hadrava, P., Yang, S., et al. 1997, A\&A, 319, 867

Hatzes, A. P., \& Cochran, W. D. 1993, ApJ, 413, 339

Hatzes, A. P., \& Cochran, W. D. 1994, ApJ, 422, 366

Horn, J., Kubát, J., Harmanec, P., et al. 1996, A\&A, 309, 521

Irwin, A. W., Campbell, B., Morbey, C. L., Walker, G. A. H., \& Yang, S. 1989, PASP, 101, 147

Keenan, P. C., \& McNeil, R. C. 1989, ApJS, 71, 245

Retter, A., Bedding, T. R., Buzasi, D. L., Kjeldsen, H., \& Kiss, L. L. 2003, ApJ, 591, L151

Smith, P. H., McMillan, R. S., \& Merline, W. J. 1987, ApJ, 317, L79

Škoda, P. 1996, in Astronomical Data Analysis Software and Systems V, ed. G. H. Jacoby, \& J. Barnes, ASP Conf. Ser., 101, 187 